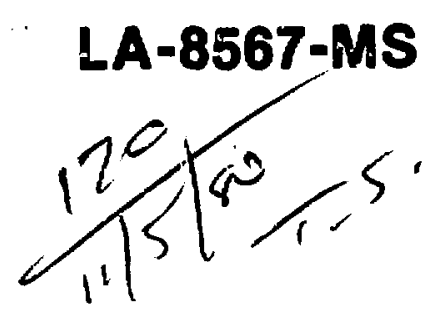

\title{
Melting Under Shock Compression
}

\author{
MASTER
}

$\frac{\pi}{\frac{\sigma}{0}}$

to

竞

ब

론 


\title{
MELTING UNDER SHOCK COMPRESSION
}

by

\author{
B. I. Bennett
}

\begin{abstract}
A simple model, using experimentally measured shock and particle velocities, is applied to the Lindemann melting formula to predict the density, temperature, and pressure at which a material will melt when shocked from room temperature and zero pressure initial conditions.
\end{abstract}

The formula for melting of a solid ${ }^{1}$ obtained by Lindemann assumed that the material would melt when the r.m.s. atomic displacement of an atom in the lattice reached $1 / 8$ of the nearest neighbor distance. This model produced the following formula for the melting temperature.

$$
T_{M}=\left[\frac{\theta A^{5 / 6}}{B \rho^{1 / 3}}\right]^{2},
$$

where $\theta$ is the Debye temperature, $A$ is the atomic weight, $\rho$ is the material density, and $B$ is a constant which varies from material to material. This constant B ranges over values of approximately 120 to 230 for a wide selection of elements. For purposes of this study, the value of $B$ will be assumed independent of material density. In particular, it will be assuned that the nelting formula will have a dependence dictated by the explicit density term appearing in the formula and the implicit dependence of the Debye temperature upon density. With these assumptions in mind, we nay write an expression for the melting temperature of a naterial undex compression. 


$$
T_{M}(\rho)=T_{M}\left(\rho_{R}\right)\left[\frac{\theta(\rho)}{\theta\left(\rho_{R}\right)} \frac{\rho_{R}^{1 / 3}}{\rho^{1 / 3}}\right]^{2},
$$

where $T_{M}\left(\rho_{R}\right)$ is the melting temperature at some reference density, $\rho_{R}$, say for conditions of zero pressure and room temperature. Written in this manner we need only concern ourselves with the variation of $\theta$ with compression and use an experimentally determined value for $T_{M}\left(\rho_{R}\right)$.

A convenient expression for the Debye temperature which encompasses three commonly used forms is given by ${ }^{2}$ :

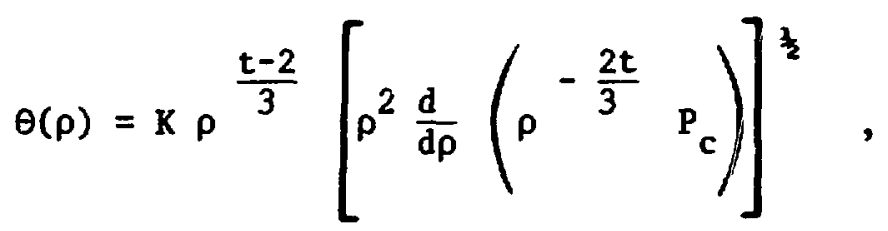

where $K$ is a constant. The parameter $t$ yields the following choices for $\theta$.

$$
t=\left\{\begin{array}{l}
0, \text { Slater-Landau expression } 3,4 \\
1, \text { Dugdale-MacDonald expression } 5 \\
2, \text { "free-volume" expression } 6
\end{array} .\right.
$$

The quantity, $P_{c}$, is the pressure obtained from the binding energy expression at $T=0$. This equation for $\theta$ produces a Grüneisen parameter, from the definition

$$
\Gamma(\rho)=\frac{\rho}{\theta(\rho)} \frac{d}{d \rho} \theta(\rho)
$$

which assymtotes to a value of $2 / 3$ (free electron gas) at large compressions and at normal solid densities gives the following relationship between the choices of the parameter $t$ for the given $P_{c}$.

$$
r_{S-I}=r_{D-M}+\frac{1}{3}=r_{f-v}+\frac{2}{3}
$$


For metals, it has been observed with some reservation ${ }^{7}$ that the Dugdale-HacDonald formula $(t=1)$ is useful for the analysis of shock data.

From Eq. (3) we find that

$$
\left.\left.\left[\frac{\theta(\rho)}{\theta\left(\rho_{R^{\prime}}\right)}\right]^{2}=\left(\frac{\rho_{R}}{\rho}\right) \frac{1 / 3}{\left[\frac{\rho d p}{d \rho}-\frac{2 t}{3} \quad P_{c}\right.}\right]_{\left[\frac{d P}{d \rho}-\frac{2 t}{3} \cdot P_{c}\right.}\right]_{\rho_{R}},
$$

allowing us, by way of Eq. (2), to express the Lindemann melting formula in terms of the density and the cold compression curve (i.e., pressure along the $\mathrm{T}=0$ isotherm).

$$
\left.T_{M}(\rho)=T_{M}\left(\rho_{R}\right) \frac{\rho_{R}}{\rho} \frac{\left[\rho \frac{d P}{d \rho}-\frac{2 t}{3} P_{c}\right.}{\left[\rho \frac{d P}{d \rho}-\frac{2 t}{3} P_{c}\right.}\right]_{\rho_{R}} .
$$

This enables us to predict the melting temperature at any compression provided that: a) The Lindemann melting formula is valid; b) The Al'tshuler expression, Eq. (3), is appropriate for Debye temperatures at compressed densities; c) We have the value of the zero temperature pressure and its derivative at the compressed density.

This latter point of knowing the cold compression curve and its derivative. has been addressed in Ref. 7 . If it is assumed that the finite temperature contribution to the pressure obeys a Mie-Grüneisen form,

$$
P-P_{c}=\rho \Gamma(\rho)\left[E-E_{c}\right] \text {, }
$$

then applying the energy and mass conservation conditions for a shocked anterial (i.e., the Hugoniot relations) ${ }^{7}$ the following equation is obtained. 


$$
{ }_{P_{H}}(\rho)=\frac{P_{c}(\rho)+\Gamma(\rho) P\left(\rho_{R}\right)\left[\frac{\rho-\rho_{R}}{2 \rho_{R}}\right]-\Gamma(\rho) \rho\left[E_{c}(\rho)-E\left(\rho_{R}\right)\right]}{1-\Gamma(\rho)\left[\frac{\rho-\rho_{R}}{2 \rho_{R}}\right]} .
$$

Here $P\left(\rho_{R}\right)$ and $E\left(\rho_{R}\right)$ are the pressure and energy of the initial state before it is shocked to a pressure $P_{H}$ and density $\rho$. Recalling that $\theta(\rho)$ and hence $\Gamma(\rho)$ can be expiessed as a functional of $P_{c}$ and its derivatives, we obtain a differential equation for the cold curve in terms of the shock pressure. Moreover, from the Hugoniot relations, we have

$$
P_{H}=\rho_{R} U_{S} U_{p},
$$

and

$$
\rho / \rho_{R}=U_{S} /\left(U_{S}-U_{p}\right)
$$

where $U_{S}$ and $U_{p}$ are the shock and particle speeds in the material.

In practice, it is observed that $U_{S}$ and $U_{p}$ for many materials are related by a linear expression.

$$
\mathrm{U}_{\mathrm{S}} \cong \mathrm{c}_{\mathrm{o}}+\mathrm{S} \mathrm{U}_{\mathrm{p}}
$$

Therefore, using the experimentally determined values of $C_{0}$ and $s, 8$ we may obtain $P_{c}(\rho)$ and $E_{c}(\rho)$.

We are now in a position to evaluate Eq. (7) to predict a melting temperature as a function of density. To determine if a material melts under shocked compression, we must be able to compute the temperature of the material along the Hugoniot curve. 
To obtain the temperature dependence of the pressure and energy we use, for the thermal contribution, the Debye model. This choice is consistent with the assumption of a Mie-Grüneisen form.

$$
\begin{aligned}
& E_{\theta}(\rho, T)=3 N_{0} k T\left[D\left(X_{D}\right)+\frac{3}{8} X_{D}\right] \\
& P_{\theta}(\rho, T)=\rho \Gamma(\rho) E_{\theta}(\rho, T)
\end{aligned}
$$

where $X_{D} \equiv \theta(\rho) / T, N_{0}$ is the number of atoms per gran of material, and $D(X)$ is the Debye integral. The total internal energy and pressure are then given by:

$$
\begin{aligned}
& E(\rho, T)=E_{c}(\rho)+E_{\theta}(\rho, T), \\
& F(\rho, T)=P_{c}(\rho)+P_{\theta}(\rho, T) .
\end{aligned}
$$

By applying the Hugoniot relations with this simple equation of state, we are able to compute the temperature along the shock pressure curve to determine the density, temperature and pressure at which the Lindemann melting law would predict the material to melt. The results for a variety of materials are presented in Table I. Also included in the table are the shock and particle velocities at this point and the predicted compression, $\eta_{M} \equiv \rho / \rho_{R}$, of the initial density.

The table entries were obtained using the Dugdale-MacDonald form of the Debye temperature. The Slater-Iaandau and "free-volume" expressions raise and lower respectively the predicted pressure at which the material melts. An additional piece of experimental data such as the bulk modulus or the Grüneisen constant could be used to deterwine the appropriate value of $t$. In fact, it could be treated as an empirical parameter with nonintegral values and correspondingly adjusted to fit such data. 
It would not be unexpected that factors such as whether or not the material underwent inelastic flow or the geometry of the shock compression could influence the outcone of experimental studies. However, if techniques could be devised to detect melting, this model might provide a test of the validity of the Iindeman formula.

\section{TABLE I}

RESULTS FOR A VARIETY OF MATERIALS

\begin{tabular}{|c|c|c|c|c|c|c|}
\hline Material & $\rho_{M}(\mathrm{gm} / \mathrm{cc})$ & $\begin{array}{l}\mathrm{T}\left({ }^{\circ} \mathbf{K}\right) \\
M \\
\end{array}$ & $\begin{array}{l}P \text { (Mbars) } \\
M \\
\end{array}$ & $\begin{array}{l}u(\mathrm{~km} / \mathrm{s}) \\
\mathrm{s} \\
\end{array}$ & $\begin{array}{l}\mathrm{U}(\mathrm{k} / \mathrm{S}) \\
\mathrm{p}\end{array}$ & $\underline{n}$ \\
\hline A1 & 4.065 & 2178 & 0.743 & 9.204 & 2.897 & 1.459 \\
\hline $\mathbf{A u}$ & 26.455 & 3652 & 1.501 & 5.349 & 1.459 & 1.375 \\
\hline $\mathrm{Be}$ & 3.113 & 3139 & 1.615 & 14.672 & 5.948 & 1.682 \\
\hline $\mathrm{Bi}$ & 13.243 & 1257 & 0.219 & 2.940 & 0.756 & 1.346 \\
\hline $\mathbf{C a}$ & 2.532 & 1600 & 0.196 & 5.707 & 2.220 & 1.637 \\
\hline$C d$ & 11.784 & 1816 & 0.450 & 4.419 & 1.179 & 1.364 \\
\hline$C_{r}$ & 10.922 & 6864 & 2.766 & 10.562 & 3.680 & 1.535 \\
\hline $\mathrm{Cu}$ & 13.271 & 4087 & 1.721 & 7.676 & 2.511 & 1.486 \\
\hline Mo & $15 \cdot 368$ & 6095 & 2.613 & 8.730 & 2.933 & 1.506 \\
\hline $\mathrm{Na}$ & 1.407 & 730 & 0.055 & 4.250 & 1.326 & 1.453 \\
\hline $\mathbf{N i}$ & 13.327 & 4938 & 2.318 & B. 842 & 2.955 & 1.502 \\
\hline $\mathbf{P b}$ & 15.185 & 1345 & 0.302 & 3.248 & 0.820 & 1.338 \\
\hline $\mathbf{S b}$ & 10.146 & 3696 & 0.463 & 4.511 & 1.532 & 1.514 \\
\hline $\mathbf{T i}$ & 7.293 & 2022 & 0.930 & 7.361 & 2.791 & 1.611 \\
\hline$W$ & 29.873 & 8125 & 3.492 & 7.138 & 2.545 & 1.554 \\
\hline
\end{tabular}




\section{REFERENCES}

1. J. M. Ziman, Electrons and Phonons, Oxford University Press, London (1963), p. 57 .

2. I. V. Al'tshuler, Soviet Physics $\underline{8}, 52$ (1965).

3. J. C. Slater, Introduction to Chemical Physics, McGraw-Hill, New York (1939), Chapter XIV.

4. L. D. Landau and K. P. Stanyrikovich, DAN SSSR 46, 399 (1945).

5. J. S. Dugdale and D. K. C. MacDonald, Phys. Rev. 89, 832 (1953).

6. Hirschfelder, Curtiss and Bird, Molecular Theory of Gases and Liquids, Wiley, New York (1954).

7. H. H. Rice, R. G. HcQueen, and J. M. Walsh, "Ccmpressicn of Solids by Strong Shock Waves," Solid State Physics, Academic Press, New York (1958), Vo1. 6, p. 1 .

8. "Selected Hugoniots," Los alamos Scientific Laboratory report IA-4167-MS (1969). 